\title{
The formation of black hole low-mass X-ray binaries: Through case $B$ or case $C$ mass transfer?
}

\author{
G. Nelemans and E. P. J. van den Heuvel ${ }^{1}$ \\ Astronomical Institute "Anton Pannekoek", Kruislaan 403, 1098 SJ Amsterdam, The Netherlands \\ Received 17 April 2001 / Accepted 27 June 2001

\begin{abstract}
The formation of low-mass X-ray binaries containing a rather massive $\left(M \gtrsim 7 M_{\odot}\right)$ black hole is problematic because in most recent stellar evolutionary calculations the immediate progenitors of these black holes (Wolf-Rayet stars) lose so much mass via their stellar wind that their final masses are well below the observed black hole masses. We discuss the recently proposed solution that these binaries are formed through case C mass transfer (i.e. mass transfer after core helium burning is completed), avoiding a long Wolf-Rayet phase and thus significant mass loss. We show that only some of the currently available models for the evolution of massive stars allow this formation channel. We also investigate the effect of the downward revised Wolf-Rayet mass-loss rate as is suggested by observations, and conclude that in that case Wolf-Rayet stars end their lives with significantly higher masses than previously found and may be able to form a black holes.
\end{abstract}

Key words. stars: statistics - binaries: close - binaries: evolution

\section{Introduction}

In low-mass X-ray binaries a neutron star or a black hole accretes from a low-mass $\left(M \lesssim 1 M_{\odot}\right)$ companion. A scenario to form such stars begins with a relatively wide binary of a massive star and a low-mass companion. When the massive star becomes a giant, mass transfer is unstable and a common-envelope forms in which the companion spirals down towards the core of the giant, leaving a close binary consisting of the helium core of the giant and the low-mass companion (van den Heuvel 1983). The helium star explodes in a supernova and depending on the (core) mass of the helium star, a neutron star or black hole is formed. With the discovery of A0620-00 (Eyles et al. 1975; Elvis et al. 1975) and the determination of the mass function of 3.18 (McClintock \& Remillard 1986), the existence of the class of black hole low-mass X-ray binaries was established. Currently we know 6 to 8 such systems depending on the membership criteria (Charles 1998; Bailyn et al. 1998). An evolutionary scenario for these objects is given in de Kool et al. (1987).

To make a black hole, the initial mass of the primary must exceed a critical value, which currently is believed to be around $20 M_{\odot}$ (Fryer 1999). However, large mass-loss rates for massive stars and Wolf-Rayet stars have been inferred from observations (e.g. de Jager et al. 1988) and are found from the comparison of Wolf-Rayet models with

Send offprint requests to: G. Nelemans, e-mail: GijsN@astro.uva.nl these observations (Langer 1989a). Applying these rates to evolutionary calculations resulted in the conclusion that even massive single stars might end their evolution as relatively low-mass objects when they explode (Schaller et al. 1992; Meynet et al. 1994; Woosley et al. 1995) and are thus unable to produce the observed black holes (see also Kalogera 1999). For massive stars in close binaries, which lose their hydrogen envelopes due to mass transfer early in their evolution the situation is even worse; the most recent calculations predict masses of helium stars as they explode as low as $3 M_{\odot}$, almost independent of their initial mass (Wellstein \& Langer 1999).

In this article we first discuss the formation of black hole low-mass X-ray binaries through case $\mathrm{C}$ evolution as suggested by Brown et al. (1999) and Wellstein \& Langer (1999): mass transfer starting after core-helium burning has been completed (Kippenhahn \& Weigert 1967). In this case a long-duration Wolf-Rayet phase in which the star loses a lot of mass is avoided (Sect. 2). Then we discuss the most recently observed mass-loss rates for WolfRayet stars and the implication of lower mass-loss rates on the final helium-star masses of exploding stars in binaries (Sect. 3). At the end we discuss uncertainties and possible alternatives for the formation of black hole low-mass X-ray binaries (Sect. 4) and end with our conclusions (Sect. 5).

\section{Case C mass transfer}

It has been suggested that case $\mathrm{C}$ mass transfer could be invoked to avoid a long-duration Wolf-Rayet phase in the 
evolution of the massive star, in order that this star does not lose too much mass and still is able to form a massive black hole (Brown et al. 1999; Wellstein \& Langer 1999).

The occurrence of case $\mathrm{C}$ mass transfer depends on the radius evolution of massive stars. For supergiants the radius of the star is not very well defined, since the outer layers of the giant envelope are extremely dilute. However, the best we can do is use the calculated values of the radii of giants. We also neglect the interaction between the wind of the massive star and the companion which may influence the separation of the two stars.

We calculate the initial separation with which a binary should start in order to undergo case C mass transfer as follows (see also Portegies Zwart et al. 1997). The separation at the moment the Roche-lobe overflow (RLOF) starts is given by

$a_{\mathrm{RLOF}}=\frac{R}{r_{\mathrm{L}}}$

where $R$ is the radius of the star and $r_{\mathrm{L}}$ is the dimensionless Roche-lobe radius (the ratio of the Roche-lobe radius and the binary separation). We use the Eggleton (1983) equation for $r_{\mathrm{L}}$. For mass ratios between 10 and 50, the value of $r_{\mathrm{L}}$ is between about 0.6 and 0.7 . During the evolution the star loses mass and the separation increases according to

$a^{\prime}=a \frac{M}{M^{\prime}}$,

where $M$ denotes the total mass of the binary. So to start Roche-lobe overflow at time $t$ when the star has a radius $R(t)$, the initial separation is given by

$$
\begin{aligned}
a_{\mathrm{i}} & =a_{\mathrm{RLOF}}(t) \frac{M(t)}{M_{\mathrm{i}}} \\
& =\frac{R(t)}{r_{\mathrm{L}}} \frac{M(t)}{M_{\mathrm{i}}} .
\end{aligned}
$$

We now compute the separations at which massive stars fill their Roche lobes as function of initial mass and initial separation and determine whether the mass transfer is case B or case C. In Fig. 1 (top) we show this for the evolutionary calculations of Schaller et al. (1992, see also Fig. 4 of Kalogera \& Webbink 1998). For a star of initially $15 M_{\odot}$ case $\mathrm{C}$ mass transfer occurs for initial separation between 1000 and $1320 R_{\odot}$. For a $20 M_{\odot}$ star, these limits are 1300 and $1550 R_{\odot}$. For a $25 M_{\odot}$ star case $\mathrm{C}$ is not possible anymore. The two other panels in Fig. 1 show the same, but for the stellar evolution models of Hurley et al. (2000, middle) and Heger et al. (2000, bottom). For these models case $\mathrm{C}$ is not possible for stars more massive than around $19 M_{\odot}$. A recent estimate of the number of black hole low-mass X-ray binaries that can form through the narrow case $\mathrm{C}$ interval of the Schaller models shows that even such a narrow interval might be enough to explain the whole Galactic population (Brown et al. 2001).

We conclude that since case $C$ evolution depends strongly on the radius evolution of massive stars which is very uncertain, it seems possible but is not certain whether

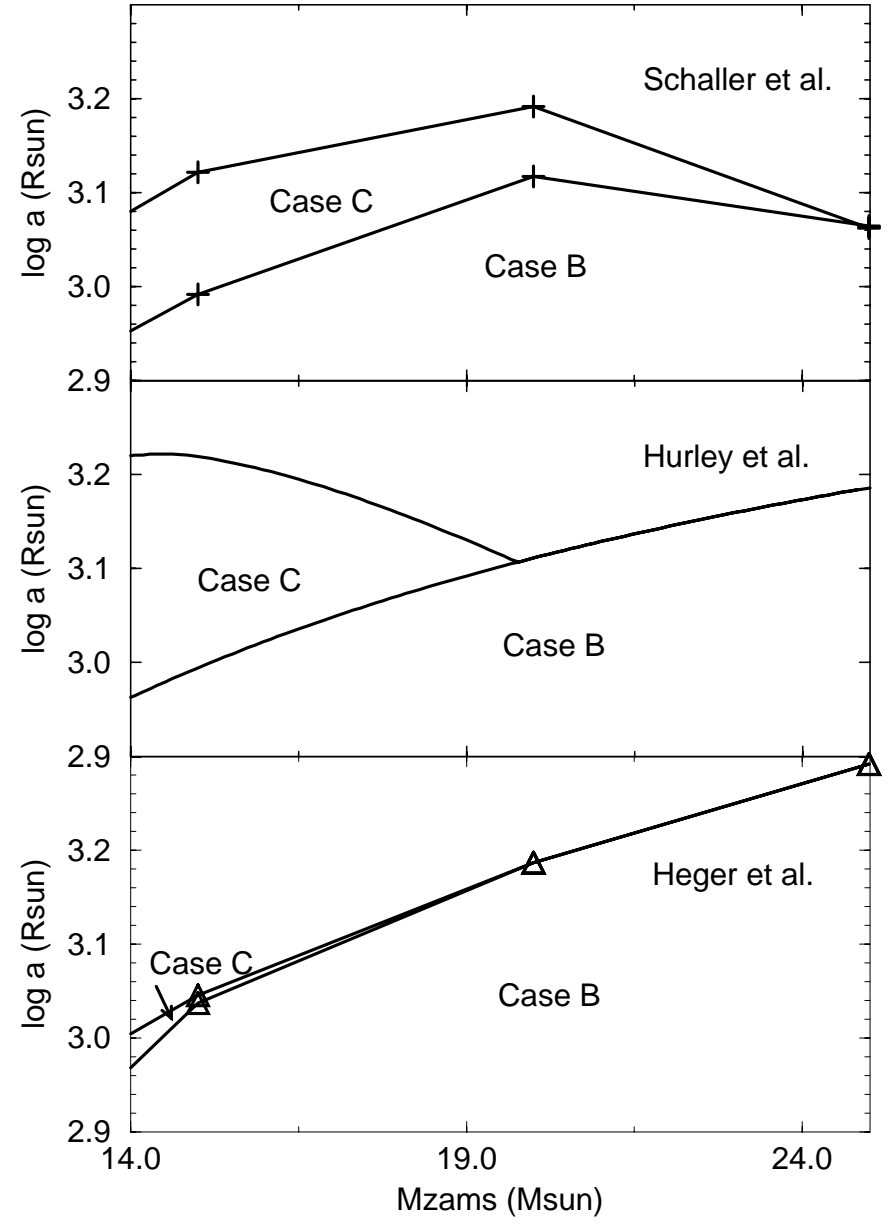

Fig. 1. Initial separations $a_{\mathrm{i}}$ for which case $\mathrm{B}$ and case $\mathrm{C}$ mass transfer occur as function of ZAMS mass, for a $1 M_{\odot}$ companion. Top for the Schaller et al. (1992) models, middle for the Hurley et al. (2000) models, bottom for the Heger et al. (2000) models.

black hole low-mass X-ray binaries can be formed in this way.

\section{Case B mass transfer}

A different way to avoid too much mass loss may be the fact that observed mass loss rates (which are the basis for the mass-loss rates used in the evolutionary calculations) are revised downward (Hamann \& Koesterke 1998; Nugis \& Lamers 2000), which may make it possible to prevent helium stars in binaries to lose so much mass they no longer can become black holes. In a recent paper with drastically lower mass-loss rates, derived from one particularly well-studied object and extrapolated, final masses over $20 M_{\odot}$ for the most massive helium stars are found (Cherepashchuk 2001). As shown by Kalogera (1999) the helium stars that were the progenitors of the black holes in binaries cannot have lost more than half of their initial mass. This includes both mass loss in the stellar wind and in the supernova explosion.

A recent compilation of observed mass-loss rates for Wolf-Rayet stars is made by Nugis \& Lamers (2000). In 


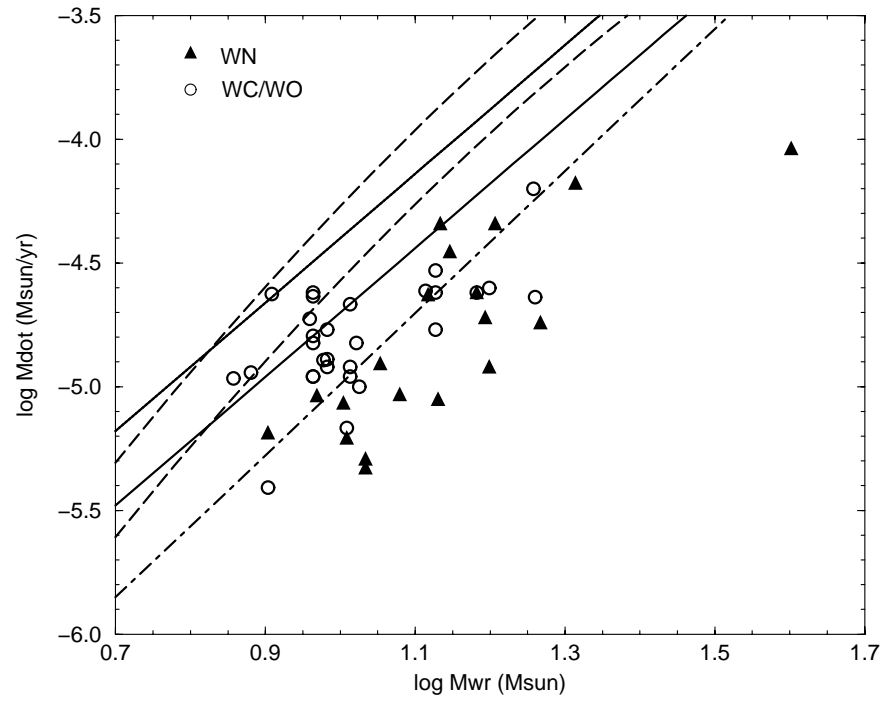

Fig. 2. Mass-loss rates for Wolf-Rayet stars as observed (triangles for $\mathrm{WN}$ and circles for WC/WO stars, from Nugis \& Lamers 2000, where we excluded the hydrogen rich Wolf-Rayet stars) and various relations used for evolutionary calculations. The solid lines are the relations assumed by Woosley et al. (1995) for WC (upper) and WN (lower) stars. The dashed lines are the ones used by Wellstein \& Langer (1999), where we converted the mass loss-luminosity relations to mass lossmass relations using the mass - luminosity relation of Langer (1989b). The upper dashed line is for their standard case, the lower for their reduced mass loss case. The dash-dotted line is a rectangular least square fit to all points (see also the text and Eq. (4)).

Fig. 2 we show these inferred mass-loss rates for WN and WC/WO stars (excluding the hydrogen rich Wolf-Rayet stars). We overplotted mass-loss rates for WN and WC stars as used by Woosley et al. (1995) as the solid lines. The mass-loss rates used recently by Wellstein \& Langer (1999) are shown as the dashed lines, where we used the luminosity-mass relation as given by Langer (1989b) to convert the mass loss-luminosity relation used by these authors, to a mass loss-mass relation. The top dashed line is their standard case, the bottom a reduced mass-loss rate, which they used to account for the lower observed mass-loss rates.

The most recently determined mass-loss rates thus suggest that the rates used by Wellstein \& Langer (1999) are still too high. We will investigate the effect of using a lower mass-loss rate law, which is shown in the figure as the dash-dotted line and is given by

$\dot{M}=-1.38 \times 10^{-8} M^{2.87}$

and is obtained by a "rectangular least square fit" (Langer 1989a) to the data (i.e. minimising the rectangular distances to the line, rather than the vertical distances). The fit is different from the one obtained by Nugis \& Lamers (2000) because we excluded the hydrogen rich Wolf-Rayet stars.

For a mass-loss rate of the form

$\dot{M}=-k M^{\alpha}$

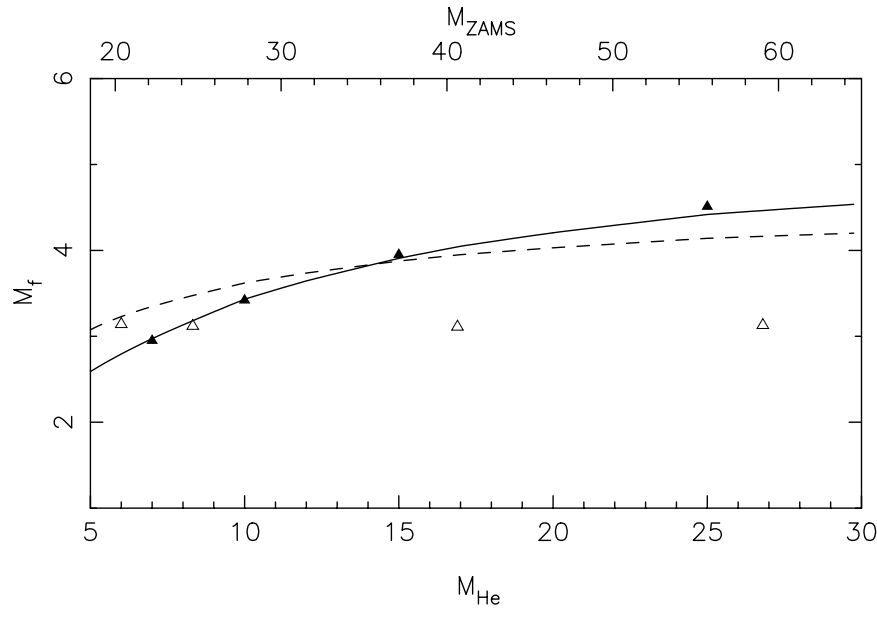

Fig. 3. Final helium star masses as function of the initial helium star mass with the mass-loss rates according to Woosley et al. (1995, solid line, some of their results are potted as solid triangles) and Wellstein \& Langer (1999, dashed line) assuming a helium star lifetime as given by Woosley et al. (1995). A selection of their results is plotted as the open triangles. The numbers at the top give an estimate of the ZAMS mass of the progenitor of the helium star.

the final helium stars mass $M_{\mathrm{f}}$ can be computed from the initial mass $M_{\mathrm{i}}$ and the helium star lifetime $(\tau)$ from

$M_{\mathrm{f}}=\left[M_{\mathrm{i}}^{1-\alpha}+(\alpha-1) k \tau\right]^{1 /(1-\alpha)}$.

As a check of our calculations we show in Fig. 3 the final masses that we obtain using the top two lines (dashed and solid) from Fig. 2 and the helium star lifetimes as given by Woosley et al. (1995) and compare these with the results obtained with the same mass-loss rates by Woosley et al. (1995) and Wellstein \& Langer (1999). For the dashed line the final mass is obtained by numerical integration of the mass evolution. The final masses do not completely agree with the masses obtained by Wellstein \& Langer (1999), probably because these high mass-loss rates lead to even longer lifetimes.

We now calculated the final masses for the revised mass-loss rate given by Eq. (4), which yields

$M_{\mathrm{f}}=\left[M_{\mathrm{i}}^{-1.87}+2.6 \times 10^{-8} \tau\right]^{-1 / 1.87}$.

In Fig. 4 we show these masses for the helium star lifetimes from Woosley et al. (1995, solid line). The lifetime of the helium star depends on the assumed mass-loss rate because mass-losing helium stars become less massive, thus less luminous and can live longer. For example the helium star lifetimes as given by Woosley et al. (1995) are substantially longer than the ones collected by Pols et al. (1991) for models without mass loss. We thus expect the lifetimes for the helium stars with reduced mass-loss rates to be shorter. In Fig. 4 we also plotted the final helium star masses assuming a lifetime which is halfway in-between the lifetimes given by Woosley et al. (1995) and Pols et al. (1991, dashed line) and the lifetime given by Pols et al. (1991, dash-dotted line). 


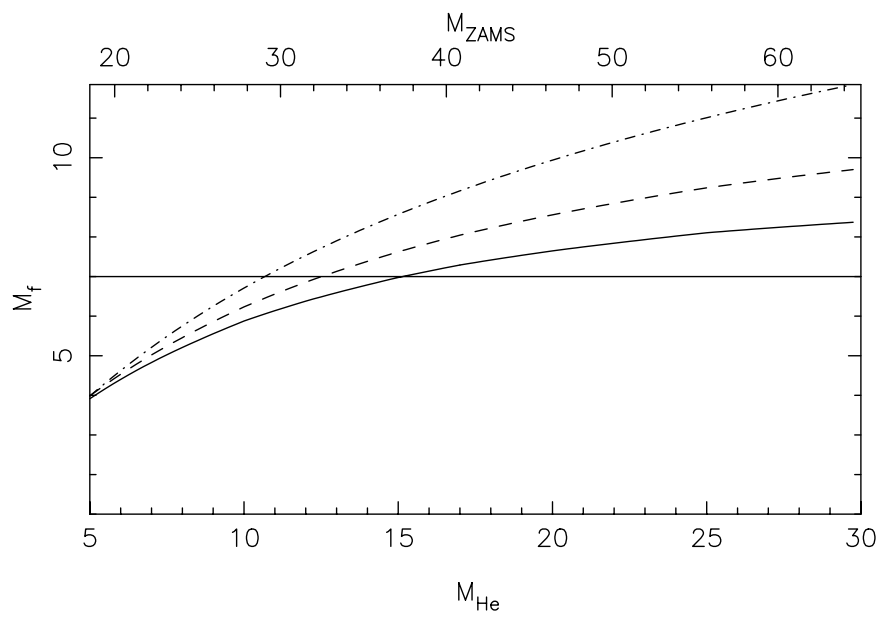

Fig. 4. Final helium star masses as function of initial helium star mass with the mass-loss rates given by Eq. (4) using a helium-star lifetime as given by Woosley et al. (1995, solid line), as given by Pols et al. (1991, dash-dotted line) and a heliumstar lifetime halfway in-between these two (dashed line). ZAMS masses for the helium star progenitors are indicated at the top.

The horizontal line is at $7 M_{\odot}$, the typical observed mass of the black holes in the low-mass X-ray binaries. The limiting ZAMS mass for which the final helium star mass exceeds $7 M_{\odot}$ with the masses loss rate used here is $\sim 30-37 M_{\odot}$.

We thus conclude that with revised mass-loss rates helium stars end their lives with significantly higher masses than previously found and may be able to form black holes even after case $B$ mass transfer.

\section{Discussion}

The analysis in Sect. 2 neglects the influence of the wind of the massive star on the companion star. The companion moves through the wind and already feels friction, which counteracts the widening of the orbit due to the stellar wind. However, even for a wind mass-loss rate of $10^{-3} M_{\odot} \mathrm{yr}^{-1}$, the density in the wind at the companion is almost four orders of magnitude lower than the density at the edge of the giant for a giant with radius of $1000 R_{\odot}$ and a binary separation of $1600 R_{\odot}$.

The whole argument presented in Sect. 3 is based on the observed mass-loss rates. However, it should be noted that all mass-loss rates proposed for Wolf-Rayet stars and used in evolutionary calculations are based on the observed rates. The valid question still remains what the uncertainty is in the observed mass-loss rates and in the inferred stellar masses and how this could influence our main conclusion.

The mass-loss rates as determined by Nugis \& Lamers (2000) are the most accurate, but still suffer from the general problem that not all quantities (mass, mass-loss rate and luminosity) can be determined independently. They therefore use the mass-luminosity relation of Schaerer \& Maeder (1992) to obtain the final mass estimates from the luminosity. Using a different mass-luminosity relation may change the resulting mass/mass-loss rate combinations.

Taking the masses and mass-loss rates as plotted in Fig. 2, one would not say that there is a unique mass-loss rate-mass relation, as is expected on theoretical grounds (Langer 1989b). The scatter is larger than the quoted uncertainty in the observations. This either points to underestimates of the errors in the observations, to variability or to additional physical processes, which were not taken into account in the calculations by Langer (1989b) and can change the mass-loss rate for a given Wolf-Rayet star mass. One could think of rotation, magnetic fields or maybe the evolutionary history.

In the last respect it might be that stars in binaries that lose their hydrogen envelopes by mass transfer evolve differently from stars that lose their envelopes due to their own stellar winds (which possibly is enhanced by a companion). The question which stars actually form black holes and which neutron stars is considerably more complex than the question of the final mass of helium stars (e.g. Fryer 1999). In particular the evolution of the core is important. As long as the collapse of the core is not understood this question will remain unanswered.

Finally, it should be noted that to form a black hole low-mass X-ray binary the companion must survive the common-envelope phase. The outcome of the common envelope depends on the binding energy and density structure of the giants envelope, which are quite different for giants that undergo case $\mathrm{B}$ and case $\mathrm{C}$ mass transfer. It could for instance be that that all binaries that undergo case B mass transfer to a low-mass companion will completely merge. That would mean that we need the small allowed initial separation range for case $\mathrm{C}$.

\section{Conclusion}

We calculated the possible initial separations for which case C mass transfer is likely to occur for binaries containing a massive star and a low-mass star, using different stellar evolution models. We find that case C mass transfer becomes impossible for primaries more massive than around $19 M_{\odot}$ for the models of Heger et al. (2000) and Hurley et al. (2000) and more massive than around $25 M_{\odot}$ for the models by Schaller et al. (1992). For such binaries either case B mass transfer occurs, or no mass transfer at all. Unless the current models for massive stars underestimate the radius expansion after the end of core helium burning the chances for forming black holes in binaries through case $\mathrm{C}$ mass transfer are therefore limited.

We also investigated the influence of the assumed mass-loss rate on the final mass of helium stars in binaries and conclude that with a downward revised mass-loss rate as suggested by the observations (e.g. Nugis \& Lamers 2000) helium stars end their lives with significantly higher masses than previously found and may be able to form black holes even after case B mass transfer for primaries more massive than $\sim 30-40 M_{\odot}$. 
Acknowledgements. We thank J. Dewi and N. Langer for helpful discussion and trial calculations of helium star evolution and A. Heger for providing details of his evolutionary calculations. This work was supported by NWO Spinoza grant $08-0$ to E. P. J. van den Heuvel.

\section{References}

Bailyn, C. D., Jain, R. K., Coppi, P., \& Orosz, J. A. 1998, ApJ, 499,367

Brown, G. E., Lee, C.-H., \& Bethe, H. A. 1999, New Astron., 4,313

Brown, G. E., Lee, C.-H., \& Tauris, T. M. 2001, New Astron., 6,331

Charles, P. A. 1998, in Theory of Black Hole Accretion Disks, ed. M. Abramowicz, G. Bjornsson, \& J. Pringle (Cambridge: CUP), 1

Cherepashchuk, A. M. 2001, ARep, 45, 120

de Jager, C., Nieuwenhuijzen, H., \& van der Hucht, K. A. 1988, A\&AS, 72, 259

de Kool, M., van den Heuvel, E. P. J., \& Pylyser, E. 1987, A\&A, 183, 47

Eggleton, P. P. 1983, ApJ, 268, 368

Elvis, M., Page, C. G., Pounds, K. A., Ricketts, M. J., \& Turner, M. J. L. 1975, Nature, 257, 656

Eyles, C. J., Skinner, G. K., Willmore, A. P., et al. 1975, IAU Circ., 2822
Fryer, C. L. 1999, ApJ, 522, 413

Hamann, W.-R., \& Koesterke, L. 1998, A\&A, 335, 1003

Heger, A., Langer, N., \& Woosley, S. E. 2000, ApJ, 528, 368

Hurley, J. R., Pols, O. R., \& Tout, C. A. 2000, MNRAS, 315, 543

Kalogera, V. 1999, ApJ, 521, 723

Kalogera, V., \& Webbink, R. F. 1998, ApJ, 493, 351

Kippenhahn, R., \& Weigert, A. 1967, ZsAp, 65, 251

Langer, N. 1989a, A\&A, 220, 135

Langer, N. 1989b, A\&A, 210, 93

McClintock, J. E., \& Remillard, R. A. 1986, ApJ, 308, 110

Meynet, G., Maeder, A., Schaller, G., Schaerer, D., \& Charbonnel, C. 1994, A\&AS, 103, 97

Nugis, T., \& Lamers, H. J. G. L. M. 2000, A\&A, 360, 227

Pols, O. R., Coté, J., Waters, F. M., \& Heise, J. 1991, A\&A, 241,419

Portegies Zwart, S. F., Verbunt, F., \& Ergma, E. 1997, A\&A, 321, 207

Schaerer, D., \& Maeder, A. 1992, A\&A, 263, 129

Schaller, G., Schaerer, D., Meynet, G., \& Maeder, A. 1992, A\&AS, 96, 269

van den Heuvel, E. P. J. 1983, in Accretion-driven stellar X-ray sources, ed. W. H. G. Lewin, \& E. P. J. van den Heuvel (Cambridge: CUP), 303

Wellstein, S., \& Langer, N. 1999, A\&A, 350, 148

Woosley, S. E., Langer, N., \& Weaver, T. A. 1995, ApJ, 448, 315 